\title{
The Kounis Syndrome, Part of MINOCA Challenge
}

\author{
Rodriguez Robert, Delgado Leal Luis*, Marin Rangel Dennise, Lugo Guadarrama Roberto, Hernandez \\ Perez Javier and Botti Sequera Arturo \\ Department of Cardiology, Mexico
}

*Corresponding author: Delgado Leal Luis, Cardiology Department, Centenario Hospital Miguel Hidalgo, Aguascalientes, Ags. Mexico

\section{ARTICLE INFO}

Received: 陆 November 07, 2019

Published: 慧 November 15, 2019

Citation: Rodriguez Robert, Delgado Leal Luis, Marin Rangel Dennise, Lugo Guadarrama Roberto, Hernandez Perez Javier, Botti Sequera Artur. The Kounis Syndrome, Part of MINOCA Challenge. Biomed J Sci \& Tech Res 22(5)-2019. BJSTR. MS.ID.003823.

Keywords: Kounis; Minoca; Stemi

\begin{abstract}
Kounis and Zavras in 1991 described the acute coronary syndrome accompanied by allergic or asthmatic state. The Kounis syndrome (KS) involves the release of cytokines by mast cells producing coronary vasospasm, erosion or rupture of the atheromatous plaque. There are different types of presentations. In the literature, there are multiple factors and drugs that are described as triggers. The treatment is challenging, since any drug can lead to anaphylaxis, patients allergic to acetylsalicylic acid and who need ACTP and stenting it is important to early desensitization approved protocols to aspirin. If anaphylaxis is present It may be necessary to use IV steroids, antihistamines, IV or SL vasodilators, epinephrine, antiaggregants and anticoagulants. The diagnosis, special approaches such as thrombus aspiration for special thinsions (hematoxylin / eosin and Giemsa) should be considered. The current international guides of STEMI put on alert of "MINOCA", to emphasize its diagnosis and treatment. The Kounis Syndrome is an entity that is still poorly understood and poorly recognized. Its suspicion, diagnosis and treatment must be spread to all medical community, in the intention to reduce the morbidity and mortality of this population.
\end{abstract}

\section{Summary}

Kounis and Zavras in 1991 described SICA accompanied by allergic or asthmatic status. Kounis syndrome involves the release of cytokines by mast cells producing coronary vasospasm, erosion or rupture of the atheromatous plaque. There are different types of presentations. In the literature, there are more and more multiple factors and drugs that are described as triggers. The treatment is challenging, since any drug can lead to anaphylaxis, in patients allergic to acetylsalicylic acid and who need stent implantation, it is important to start aspirin desensitization early with approved protocols. When anaphylaxis occurs, the use of IV steroids, antihistamines, IV or SL vasodilators, epinephrine, antiplatelet agents and anticoagulants may be necessary. For its diagnosis, special considerations should be made such as thrombus aspiration to perform special tinsions, (hematoxylin / eosin and Giemsa). The current international IAM CEST guides put "MINOCA" on alert, thereby emphasizing their diagnosis and treatment. Kounis syndrome is an entity that is still poorly understood, and poorly recognized, its suspicion, diagnosis and treatment must be known to the medical community to reduce the morbidity and mortality of that population.

\section{Introduction}

Current guidelines for Acute Myocardial Infarction with STsegment elevation (STEMI) alert the causes of Myocardial Infarction whit Non-Obstructive Coronary Arterial Disease (MINOCA) and the need for its clarification, to emphasize its treatment [1]. Kounis and Zavras in 1991 described for the first time the acute coronary syndrome that is accompanied with the allergic or asthmatic state. The mechanism of KS involves the release of inflammatory cytokines through the activation of mast cells which produces coronary arteries vasospasm, as well erosion or rupture of the coronary atheromatous plaque or newly described stent thrombosis [2-6]. The prevalence is still not well known, it's have been observed 
great variations between countries, being greater where this entity is suspected. The possibility of suffering an allergic reaction in life is $1.6 \%$, and of these the percentage that could develop KS is still not well established [4]. It is presented below an uncommon clinical entity in its typical presentation. The so-called "Allergic acute coronary syndrome" [2-4]

\section{Case Report}

46 years old female, 20 years of asthma history, and Fernand Widal Syndrome which consist in a triade of allergic rhinitis, allergy to acetylsalicylic acid in addition to nasal polyposis, diagnosis perform by otorhinolaryngologist $[7,8]$. It does not have cardiovascular risk factors. She started one month before admission with low intensity angina, associated with bronchospasm and allergic rhinitis exacerbation. She came to emergency service after suffer intense angina associated with bronchospasm plus adrenergic discharge, electrocardiographic changes were observed; anteroseptal lesion and inferior subepicardial ischemia where noticed. (Figure 1) STEMI associated with Fernand Widal Syndrome was diagnosed, which aroused the suspicion of probable Kounis Syndrome. She was taken to primary angioplasty where the right radial artery was approached without complications during the procedure, no allergic reactions to contrast agent. Multiple projections of the entire coronary anatomy were made. No angiographic lesions were found (Figures $2 \& 3$ ). Laboratory tests revealed positive levels of Troponin I HS $3.67 \mathrm{ng} / \mathrm{ml}$. The Thorax radiograph did not demonstrate pathology (Figure 4). Subsequently, she was admitted to coronary care unit, where she develop new episode of bronchospasm, associated with angina and new electrocardiographic changes (Figure 5). This clinically demonstrates the coronary spasm during exacerbation of asthma. Once bronchospasm crisis was controlled, no further angina, electrocardiographic changes or elevation of bio-markers from myocardial lesion were observed after.

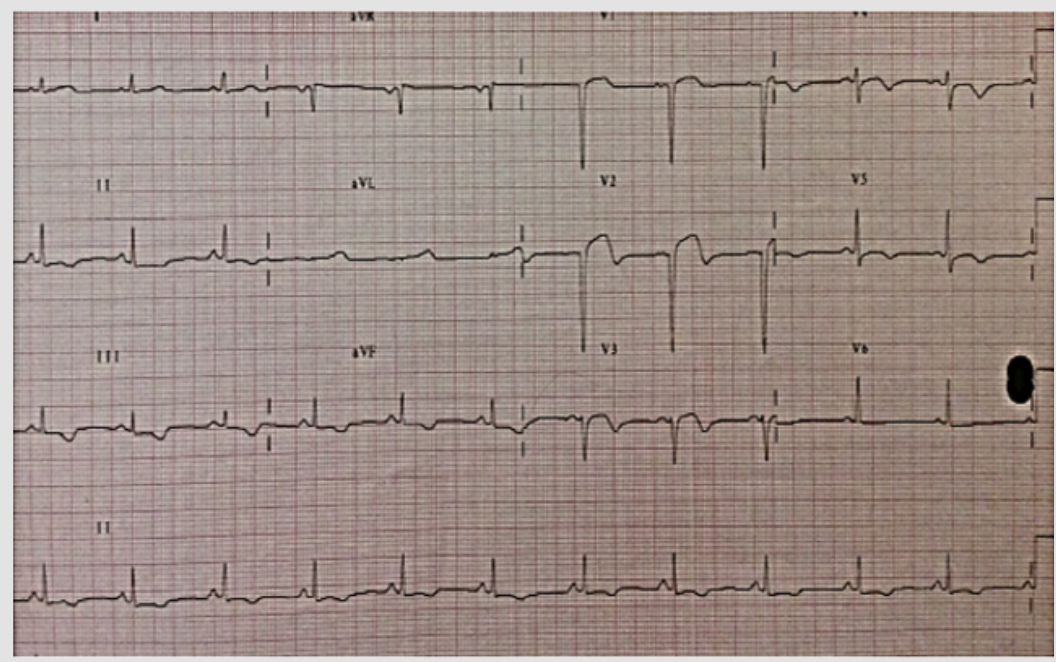

Figure 1: EKG at the emergency department.

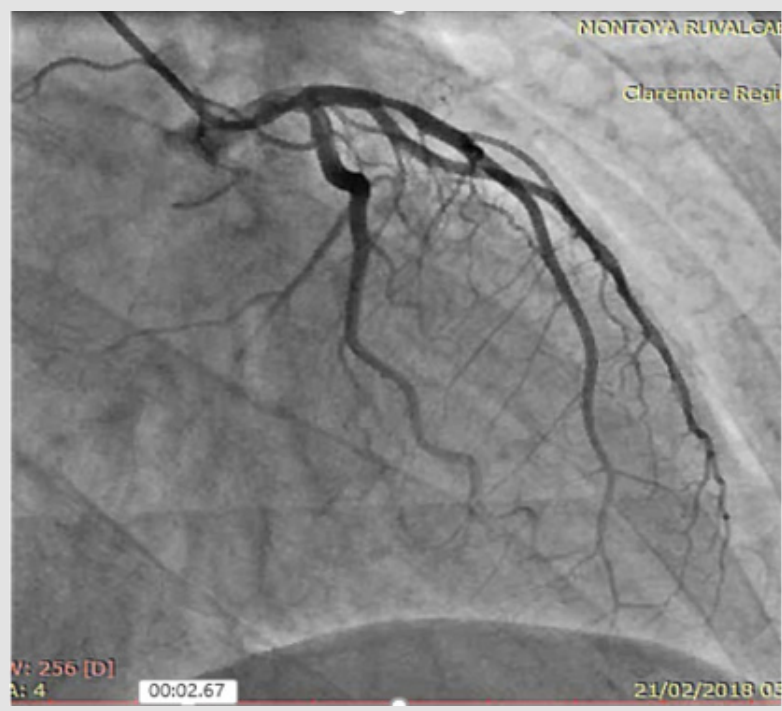

Figure 2: Angiocoronarography without disease. 


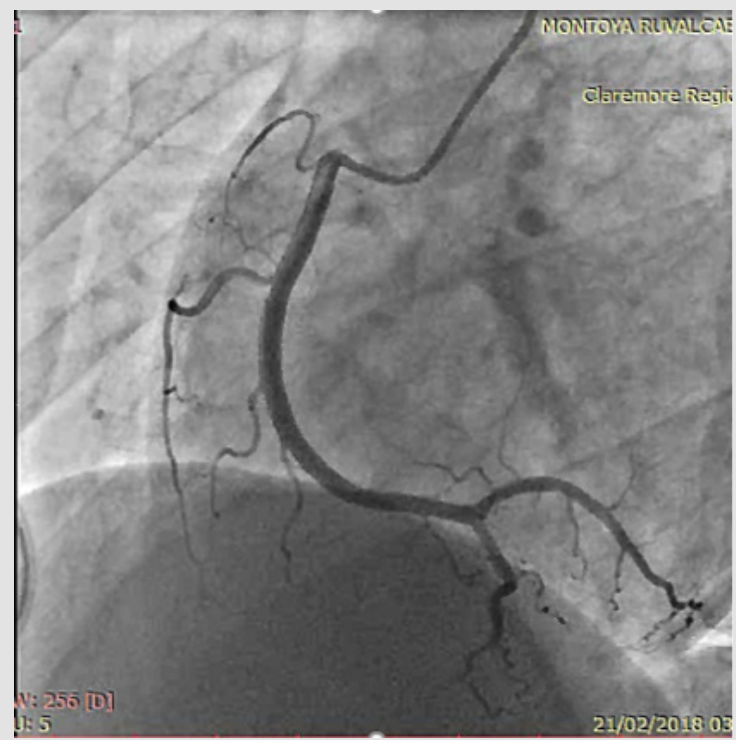

Figure 3: Angiocoronarography without disease.

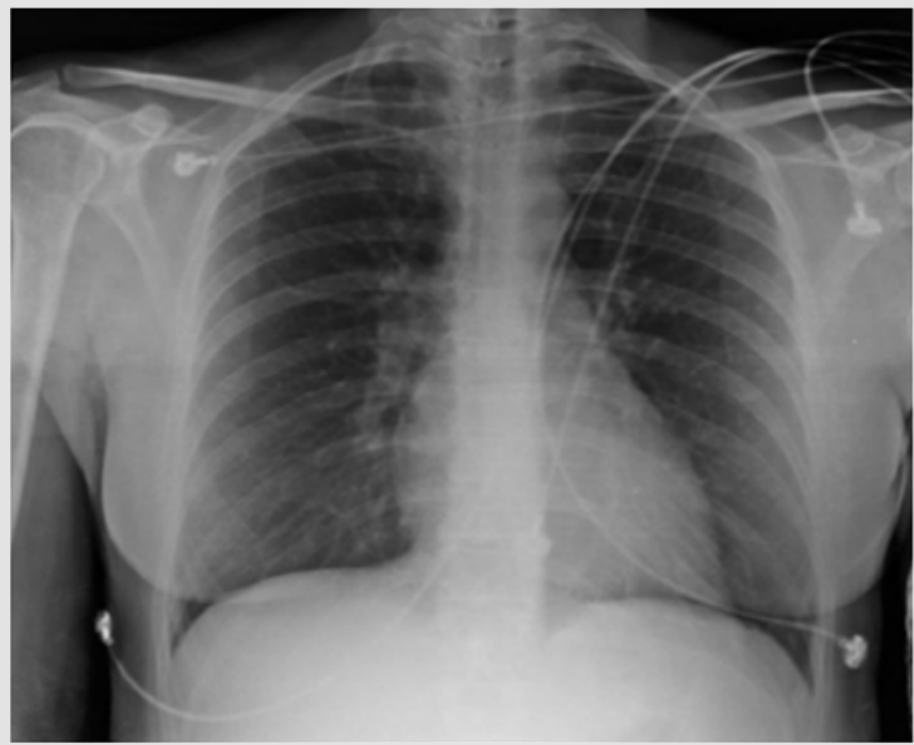

Figure 4: Chest x-ray.

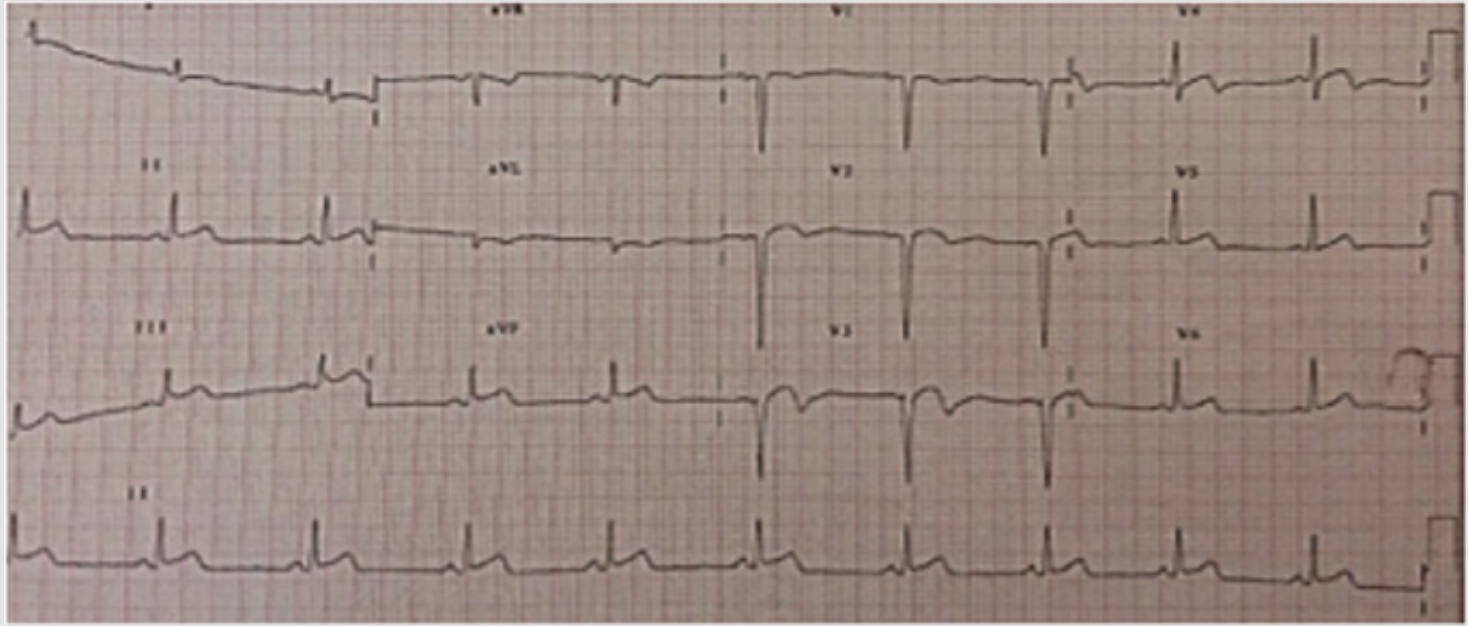

Figure 5: EKG with new ST elevation during asthmatic crisis, shows different location of myocardial lesion. 


\section{Entity}

The pathophysiology involves the mast cell activation trough inflammatory mediators such products of arachidonic acid, histamine, proteases, cytokines, chemokines, platelet activator factor, this activation began locally (stent) or in a systemic way. Currently it has been noticing that the mast cell itself can lead of enhance $\mathrm{T}$ cell activation and in that way proliferation of mast cells, these activate macrophages, and these activate $\mathrm{T}$ cells, leading to vicious circle. 4 Been in a hypersensitivity state lead to degranulation of mast cell, histamine can induce coronary spasm. The proteases tryptase, chymase, cathepsin-D can activate matrix metalloproteinases causing erosion or rupture of the fibrous cap [4]. Recently a new classification has been established, The KS now has become a

Table 1: Factors related to induce Kounis Syndrome. bigger entity and the adequate typification need to be done correctly. The KS Type I includes patients with normal or nearly normal coronary arteries without predisposing factors for coronary artery disease in whom acute allergic attacks can induce ischemic or Myocardial infarction [2-4]. KS Type II includes patients with culprit but quiescent pre-existing atheromatous disease in whom the allergic state and the mast cell degranulation lead to coronary artery spasm, plaque erosion or rupture manifesting as acute myocardial infarction [4]. Recently a new type of KS was described, Type III presents as stent thrombosis in whom thrombus harvesting and staining with hematoxylin-eosin and Giemsa reveals the presence of eosinophils and mast cells in the specimen [4-7]. This syndrome has been related to certain environmental exposures, insects and drugs of which there is a great variety (Table 1 ).

\begin{tabular}{|c|c|c|}
\hline Drugs & Conditions & Other Factors \\
\hline $\begin{array}{c}\text { Antibiotics: ampicillin, amoxicillin, amikacin, cephalosporin's, Trimethoprim- } \\
\text { sulfamethoxazole, vancomycin. }\end{array}$ & Asthma. & Latex Contact. \\
Analgesics and NSAI: aspirin, ibuprofen, dipyrone, diclofenac, naproxen. \\
$\begin{array}{c}\text { Anesthetics and others agents: midazolam, alprazolam, etomidate, isoflurane, } \\
\text { propofol, rocuronium, succinylcholine. }\end{array}$ & Fernand Widal Syndrome. \\
Anticoagulants: LMWH, UFH & Food allergy & Pets. \\
Intracoronary stenting & Mastocytosis. & Jellyfish sting \\
Contrast agents: sodium indigotindisulfonato, loxagate, lohexone. & Poison ivy \\
Glucocorticoids: betamethasone, hydrocortisone. & Viper venom \\
Others: insulin, enalapril, esmolol, protamine, allopurinol. & & \\
\hline
\end{tabular}

The drugs mainly related to analgesics, antibiotics and contrast media [4]. The treatment of this entity is supposed to be challenging, since it can present up to anaphylaxis [1-4]. Type I KS can be treated with rapid-acting IV corticosteroids (hydrocortisone $5 \mathrm{mg} / \mathrm{kg} /$ dose) and H1 IV antihistamines, IV or SL, vasodilators are safe as long as adequate arterial pressure is maintained, calcium channels blockers can stop vasospasm, as long as there is no contraindication for its use. The KS Type II, in addition to the a fore mentioned, must initiate the anti-platelet and anticoagulant treatment for STEMI. If a long lasting therapy is planned in an allergic patient to aspirin there is a safe protocol to desensitization, if the patient needs a primary PCI, the desensitization can be start after PCI, in a NSTEMI in who PCI is planned, the protocol begin before PCI using the ADAPTED protocol using a $330 \mathrm{~min}$ dead line to ensure desensitization [9]. When some variant of KS presents with anaphylaxis the risk of death increase, it should be evaluated carefully since the usual medication used in anaphylaxis can worse the hemodynamic state, in that scene, epinephrine without sulfite is preferable and applied IM or SC (dose of $0.2-0.5 \mathrm{mg}$ ). In patients chronically treated with Beta Blockers, the effect of epinephrine can be attenuated, Glucagon is adequate ( 1 to $5 \mathrm{mg}$ per IV / $5 \mathrm{~min}$ ), followed by an infusion of 5 to $15 \mu \mathrm{g} /$ min.12 Opioids administered to reduce acute pain, such as morphine, should be administered with caution in KS since allergic reactions can occur [2]. In the variant of KS Type III, in addition to carrying out the acute coronary syndrome protocol, intrastent thrombus aspiration should be performed to ensure histological examination of the material aspirated for staining in search of eosinophils (hematoxylin / eosin) and mast cells (Giemsa) [6,7,11].

\section{Conclusion}

As it has been reviewed in the literature, this particular syndrome must reach the knowledge of every physician related to these two "allergic and ischemic" entities, in order to increase their clinical recognition and ensure the best therapeutic option. Patients with an aspirin allergic history, who suffers from an acute coronary syndrome lead to PCI, should be considered and appropriately treated and desensitized.

\section{References}

1. Ibanez B, James S, Agewall S, Antunes MJ, Bucciarelli-Ducci C, et al. (2018) 2017 ESC Guidelines for the management of acute myocardial infarction in patients presenting with ST-segment elevation: The Task Force for the management of acute myocardial infarction in patients presenting with ST-segment elevation of the European Society of Cardiology (ESC). Eur Heart J 39(2): 119-177.

2. Kounis NG (2006) Kounis syndrome (allergic angina and allergic myocardial infarction): a natural paradigm?. Int J Cardiol 110(1): 7-14.

3. Sarfaraz Memon, Lovely Chhabra, Shihab Masrur, Matthew W Parker (2015) Allergic acute coronary syndrome Kounis syndrome. Proc (Bayl Univ Med Cent) 28(3): 358-362. 
4. Kounis NG, Mazarakis A, Tsigkas G, Giannopoulos S, Goudevenos (2011) Kounis syndrome: A new twist on an old disease. Future Cardiol 7(6): 805-824.

5. Chen JP, Hou D, Pendyala L, Goudevenos JA, Kounis NG (2009) Drugeluting stent thrombosis: the Kounis hypersensitivity-associated acute coronary syndrome revisited. JACC Cardiovasc Interv 2(7): 583-593.

6. Almpanis G, Mazarakis A, Tsigkas G, Koutsojannis C, Kounis GN (2010) Acute stent thrombosis and atopy: implications for Kounis syndrome. Int J Cardiol 145(2): 398-400.

7. Kogias JS, Papadakis EX, Tsatiris CG, Hahalis G, Kounis GN, et al. (2010) Kounis syndrome: a manifestation of drug-eluting stent thrombosis associated with allergic reaction to contrast material. Int J Cardiol 139(2): 206-209.

8. Ndiaye M, Hane AA, Ndir M, Ba O, Cissokho S, et al. (1999) Fernand Widal Syndrome: Apropos of 2 cases. Dakar Med 44(2): 232-235.

ISSN: 2574-1241

DOI: 10.26717/BJSTR.2019.22.003823

Delgado Leal Luis. Biomed J Sci \& Tech Res

cC) (i) This work is licensed under Creative

Submission Link: https://biomedres.us/submit-manuscript.php
9. Roberta Rossini, Annamaria Iorio, Roberto Pozzi, Matteo Bianco, Giuseppe Musumeci, et al. (2017) Aspirin desensitization in patients with coronary artery disease: results of the multicenter ADAPTED registry (Aspirin Desensitization in patients with coronary artery disease). Circ Cardiovasc Interv 10(2).

10. Cordoba-Soriano JG, Corbi-Pascual M, Lopez-Neyra I, Navarro-Cuartero J, Hidalgo-Olivares V, et al. Early aspirin desensitization in unstable patient with acute coronary syndrome: Short and long-term efficacy and safety. Eur Heart J Acute Cardiovasc Care 5(7): 41-50.

11. Nicholas G Kounis (2016) Mast Cells And The Heart: Kounis Syndrome And Takotsubo Cardiomyopathy. The Mastocytosis society.

$\begin{array}{ll}\text { BIOMEDICAL } & \text { Assets of Publishing with us } \\ \text { RESEARCHES } & \text { - Global archiving of articles } \\ & \text { - Immediate, unrestricted online access } \\ & \text { - Rigorous Peer Review Process } \\ \end{array}$

